\title{
THE INFLUENCE OF TERMINAL APPARATUS ON TELEPHONIC TRANSMISSION.
}

\author{
By Louis Cohen.
}

The problem of telephonic transmission has been very ably treated by several investigators. One has only to examine the collected papers of Dr. Oliver Heaviside and the two papers on the Propagation of Electrical Waves by Prof. M. I. Pupin to find a discussion of almost every phase of the problem. But while there is very little that one could add to the mathematical treatment of the problem, yet there is one point in connection with this problem, "The Influence of Terminal Apparatus on Telephonic Transmission," which has not been given sufficient attention, and I trust a brief discussion of this phase of the problem will be of some interest.

The only treatment of the problem that I am familiar with, that takes into account the effect of the terminal apparatus, is that by Dr. O. Heaviside: "Effect of a periodic impressed force acting at one end of a telegraphic circuit with any terminal condition." ${ }^{1}$ The derivation, however, of the formula as given in this paper is not very easy to follow, and the expressions he obtains for the current and potential along the line are of a very complicated form, and therefore do not make apparent the detrimental effect of the terminal apparatus.

In general whatever influence the line may have in distorting a telephonic wave, when the wave reaches the receiving instrument part of it will be reflected, and will merely dissipate itself on the line, and part will be absorbed and furnish the energy necessary to operate the instrument. Either portion of the wave, however, be it the part reflected or absorbed, is a function of the frequency, and therefore every harmonic will be affected differently, which will

\footnotetext{
${ }^{1}$ Collected papers, vol. 2 , p. 245 .
} 
necessarily produce a certain amount of distortion. The extent of the distortion that the wave will suffer in passing through the receiving instrument may under certain circumstances be considerable.

The final expressions obtained in this paper for the current and potential at the end of the line are. of very simple form and convenient for numerical calculations, enabling us to calculate the extent of the distortion produced by the terminal apparatus.

An examination of the final expressions (20) will, I think, make apparent at once the effect of the receiver on the distortion. Certain improvements which suggest themselves will be considered in the latter part of this paper.

Let $L, R, C$ denote the inductance, resistance, and capacity per unit length of line; let also $L_{0}, R_{0}, C_{0}$ and $L_{1}, R_{1}, C_{1}$ denote the inductance, resistance, and capacity of the sending and receiving instruments, respectively. Assume also that the leakage conductance of the line is negligible. Denoting by $X$ the current at any point on the line and $s$ the distance from the sending end, which we shall consider as the origin, then we shall have for any point on the line

$$
L \frac{d x}{d t}+R x=-\frac{d v}{d s}
$$

which expresses the fact that the algebraic sum of the electromotive forces acting at any point is zero. We also have the relation

$$
C \frac{d v}{d t}=-\frac{d x}{d s}
$$

From equations (I) and (2) we get the differential equation of propagation, now commonly known as the "telegraphist equation," which is as follows:

$$
L \frac{d^{2} x}{d t^{2}}+R \frac{d x}{d t}=\frac{I d^{2} x}{C} \frac{d s^{2}}{d}
$$

If the current is simple harmonic with respect to time, we may put

$$
X=x^{\prime} e^{i p t}
$$

and equation (3) becomes

$$
\left(-C L p^{2}+R i p C\right) x^{\prime}=\frac{d^{2} x^{\prime}}{d s^{2}}
$$


The complete solution of equation (4) is

$$
x^{\prime}=A \sin \mu s+B \cos \mu s
$$

and therefore

$$
X=\{A \sin \mu s+B \cos \mu s\} e^{i p t}
$$

Introducing the value of $X$ as given by (5) into equation (4), we find that

$$
-\mu^{2}=-C L p^{2}+R i p C
$$

Putting $\mu=a+i \beta$ and then equating the reals and imaginaries separately, we shall get

$$
\begin{aligned}
& a=\sqrt{\frac{\mathrm{I}}{2}} \sqrt{C p\left\{\sqrt{p^{2} L^{2}+R^{2}+p L}\right\}} \\
& \left.\beta=\sqrt{\frac{\mathrm{I}}{2}} \sqrt{C p\left\{\sqrt{p^{2} L^{2}+R^{2}}-p L\right.}\right\}
\end{aligned} .
$$

The value of $V$, the potential at any point on the line, can be obtained from (6) by the aid of equation (2).

We thus find

$$
V=\frac{\mu}{C i p}\{B \sin \mu s-A \cos \mu s\} e^{i p t}
$$

The constants $A$ and $B$ in equations (6) and (8) must be determined from the boundary conditions.

When

$$
\begin{aligned}
s & =l \\
V_{l} & =X_{l}\left\{R_{1}+i\left(P L_{1}-\frac{\mathrm{I}}{C_{1} p}\right)\right\}
\end{aligned}
$$

Introducing the values of $V_{l}$ and $X_{l}$, we get $\underset{C_{i p}}{\mu}\{B \sin \mu l-A \cos \mu i\}=\left\{R_{1}+i\left(L_{1} p-\frac{I}{C_{1} p}\right)\right)\{A \sin \mu l+B \cos \mu l\}$

or

$$
\{B \sin \mu l-A \cos \mu l\}=\left(H_{1}+i F_{1}\right)\{A \sin \mu l+B \cos \mu l\}
$$


where

$$
H_{1}+i F_{1}=\frac{C i p}{\mu}\left\{R_{1}+i\left(L_{1} p-\frac{\mathrm{I}}{C_{1} p}\right)\right\}
$$

Equating reals and imaginaries separately, we get

$$
\begin{gathered}
H_{1}=\frac{C p}{a^{2}+\beta^{2}}\left\{-a\left(L_{1} p-\frac{\mathrm{I}}{C_{1} p}\right)+\beta R_{1}\right\} \\
F_{1}=\frac{C p}{a^{2}+\beta^{2}}\left\{\beta\left(L_{1} p-\frac{\mathrm{I}}{C_{1} p}\right)+a R_{1}\right\}
\end{gathered}
$$

When $s=0$ we have

$$
V_{0}=E e^{i p t}-X_{0}\left\{R_{0}+i\left(L_{0} p-\frac{\mathrm{I}}{C_{0} p}\right)\right\}
$$

$E e^{i p t}$ is the impressed electromotive force.

Introducing the values of $V_{0}$ and $X_{0}$, we get

$$
\begin{aligned}
& \frac{-\mu A}{C i p}=E-B\left\{R_{0}+i\left(L_{0} p-\frac{\mathrm{I}}{C_{0} p}\right)\right\} \\
& \frac{E C i p}{\mu}=\frac{B C i p \mid}{\mu}\left\{R_{0}+i\left(L_{0} p-\frac{\mathrm{I}}{C_{0} p}\right)\right\}-A
\end{aligned}
$$

or

$$
E \frac{C i p}{\mu}=\left(T_{1}+i T_{2}\right) B-A
$$

where

$$
\begin{aligned}
& T_{1}=\frac{C P}{a^{2}+\beta^{2}}\left\{-a\left(L_{0} p-\frac{\mathrm{I}}{C_{0} p}\right)+\beta R_{0}\right\} \\
& T_{2}=\frac{C p}{a^{2}+\beta^{2}}\left\{\beta\left(L_{0} p-\frac{\mathrm{I}}{C_{0} p}\right)+a R_{0}\right\}
\end{aligned}
$$

From (9) and (II) we can determine the value of the constants $A$ and $B$, which are as follows:

$$
\begin{aligned}
& A=\frac{E C i p\left\{\sin \mu l-\left(H_{1}+i F_{1}\right) \cos \mu l\right\}}{\mu\left(T_{1}+i T_{2}\right)\left\{\left(H_{1}+i F_{1}\right) \sin \mu l+\cos \mu l\right\}-\mu\left\{\sin \mu l-\left(H_{1}+i F_{1}\right) \cos \mu l\right\}} \\
& B=\frac{E C i p\left\{\cos \mu l+\left(H_{1}+i F_{1}\right) \sin \mu l\right\}}{\mu\left(T_{1}+i T_{2}\right)\left\{\left(H_{1}+i F_{1}\right) \sin \mu l+\cos \mu l\right\}-\mu\left\{\sin \mu l-\left(H_{1}+i F_{1}\right) \cos \mu l\right\}}
\end{aligned}
$$


Introducing these values of $A$ and $B$ in equations (6) and (8) we get

$$
X=\frac{E C i p\left\{\cos \mu(l-s)+\left(H_{1}+i F_{1}\right) \sin \mu(l-s)\right\} e^{i p t}}{\mu\left(T_{1}+i T_{2}\right)\left\{\left(H_{1}+i F_{1}\right) \sin \mu l+\cos \mu l\right\}-\mu\left\{\sin \mu l-\left(H_{1}+i F_{1}\right) \cos \mu l\right\}}
$$$$
V=\frac{E \mu\left\{\left(H_{1}+i F_{1}\right) \cos \mu(l-s)-\sin \mu(l-s)\right\} e^{i \not t}}{\mu\left(T_{1}+i T_{2}\right)\left\{\left(H_{1}+i F_{1}\right) \sin \mu l+\cos \mu l\right\}-\mu\left\{\sin \mu l-\left(H_{1}+i F_{1}\right) \cos \mu l\right\}}
$$

Remembering now that $\mu=a+i \beta$, we call write

$$
\left.\begin{array}{l}
\sin \mu l=\frac{\mathrm{I}}{2} \sin a l\left(e^{\beta l}+e^{-\beta l}\right)+\frac{i}{2} \cos a l\left(e^{\beta l}-e^{-\beta l}\right) \\
\cos \mu l=\frac{\mathrm{I}}{2} \cos a l\left(e^{\beta l}+e^{-\beta l}\right)-\frac{i}{2} \sin a l\left(e^{\beta l}-e^{-\beta l}\right)
\end{array}\right\}
$$

Introducing the above values of $\sin \mu l$ and $\cos \mu l$ in the denominators of equations (I4) and (I 5) and then separating the reals and imaginaries, we shall have

$$
\begin{gathered}
\mu\left(T_{1}+i T_{2}\right)\left\{\left(H_{1}+i F_{1}\right) \sin \mu l+\cos \mu l\right\}-\mu\left\{\sin \mu l-\left(H_{1}+i F_{1}\right) \cos \mu l\right\}= \\
K_{1}+i K_{2}
\end{gathered}
$$

where

$$
\begin{aligned}
& K_{1}=\left(\frac{e^{\beta l}+e^{-\beta l}}{2}\right)\left(T_{1} a \cos a l-T_{2} \beta \cos a l-a \sin a l\right) \\
& +\left(\frac{e^{\beta l}+e^{-\beta l}}{2}\right)\left\{-C p \cos a l\left(L_{1} p-\frac{\mathrm{I}}{C_{1} p}\right)-T_{2} C p R_{1} \sin a l\right\} \\
& \quad-T_{1} C p\left(L_{1} p-\frac{\mathrm{I}}{C_{1} p}\right) \sin a l \\
& +\left(\frac{e^{\beta l}-e^{-\beta l}}{2}\right)\left(C p R_{1} \sin a l-T_{1} C p R_{1} \cos a l\right. \\
& \left.+T_{2} C p\left(L_{1} p-\frac{\mathrm{I}}{C_{1} p}\right) \cos a l\right) \\
& +\left(\frac{e^{\beta l}-e^{-\beta l}}{2}\right)\left(T_{1} \beta \sin a l+T_{2} a \sin a l+\beta \cos a l\right)
\end{aligned}
$$


and

$$
\begin{gathered}
K_{2}=\left(\frac{e^{\beta l}+e^{-\beta l}}{2}\right)\left(-T_{1} \beta \cos a l+T_{2} a \cos a l-\beta \sin a l\right) \\
+\left(\frac{e^{\beta l}-e^{-\beta l}}{2}\right)\left(C p R_{1} \cos a l+C p R_{1} T_{1} \sin a l\right. \\
\left.-T_{2} C p\left(L_{1} p-\frac{\mathrm{I}}{C_{1} p}\right) \sin a l\right) \\
+\left(\frac{e^{\beta l}-e^{-\beta l}}{2}\right)\left\{C p\left(L_{1} p-\frac{\mathrm{I}}{C_{1} p}\right) \sin a l-T_{2} C p R_{1} \cos a l\right\} \\
\quad-T_{1} C p\left(L_{1} p-\frac{\mathrm{I}}{C_{1} p}\right) \cos a l \\
+\left(\frac{e^{\beta l}-e^{-\beta l}}{2}\right)\left(-T_{1} a \sin a l+T_{2} \beta \sin a l-a \cos a l\right)
\end{gathered}
$$

If we neglect the constants of the transmitting apparatus, then $T_{1}=0$ and $T_{2}=0$, and consequently the expressions for $K_{1}$ and $K_{2}$ will be greatly simplified, thus:

$$
\begin{gathered}
K_{1}=\left(\frac{e^{\beta l}+e^{-\beta l}}{2}\right)\left(-a \sin a l-C p\left(L_{1} p-\frac{\mathrm{I}}{C_{1} p}\right) \cos a l\right)+\left(\frac{e^{\beta l}+e^{-\beta l}}{2}\right) \\
\left(\beta \cos a l+C p R_{1} \sin a l\right) \\
K_{2}=\left(\frac{e^{\beta l}+e^{-\beta l}}{2}\right)\left(-\beta \sin a l+C p R_{1} \cos a l\right)+\left(\frac{e^{\beta l}-e^{-\beta l}}{2}\right)(-a \cos a l \\
\left.+C p\left(L_{1} p-\frac{\mathrm{I}}{C_{1} p}\right) \sin a l\right)
\end{gathered}
$$

If we put for convenience $l-s=\xi$ and then substitute for $\cos \mu \xi$ and $\sin \mu \xi$ their values as given by equation (I6), we shall have

$$
X=\frac{E C i p\left(i K_{1}+K_{2}\right)}{2\left(K_{1}^{2}+K_{2}^{2}\right)}\left\{\cos a \xi\left(e^{\beta \xi}+e^{-\beta \xi}\right)-i \sin a \xi\left(e^{\beta \xi}-e^{-\beta \xi}\right)+\right.
$$

$H_{1} \sin a \xi\left(e^{\beta \xi}+e^{-\beta \xi}\right)+i H_{1} \cos a \xi\left(e^{\beta \xi}-e^{-\beta \xi}\right)+i F_{1} \sin a \xi\left(e^{\beta \xi}+e^{-\beta \xi}\right)$

$\left.-F_{1} \cos \alpha \xi\left(e^{\beta \xi}-e^{-\beta \xi}\right)\right\} e^{i p t}$ 


$$
\begin{aligned}
V= & \frac{E(a+i \beta)\left(K_{1}-i K_{2}\right)}{2\left(K_{1}^{2}+K_{2}^{2}\right)}\left\{H_{1} \cos a \xi\left(e^{\beta \xi}+e^{-\beta \xi}\right)-i H_{1} \sin a \xi\left(e^{\beta \xi}-e^{-\beta \xi}\right)\right. \\
& +i F_{1} \cos a \xi\left(e^{\beta \xi}+e^{-\beta \xi}\right)+F_{1} \sin a \xi\left(e^{\beta \xi}-e^{-\beta \xi}\right)-\sin a \xi\left(e^{\beta \xi}+e^{-\beta \xi}\right) \\
& \left.-i \cos a \xi\left(e^{\beta \xi}-e^{-\beta \xi}\right)\right\} e^{i p t}
\end{aligned}
$$

After some simplification and a few reductions the real part of the above two expressions will be as follows:

$$
\begin{gathered}
X=\frac{E C p}{2 \sqrt{K_{1}^{2}+K_{2}^{2}}}\left\{\left[e^{\beta \xi} \cos (p t-a \xi+\theta)+\right.\right. \\
\left.+e^{-\beta \xi} \cos (p t+a \xi+\theta)\right]+\sqrt{H_{1}^{2}+F_{1}^{2}}\left[-e^{\beta \xi} \cos \left(b t-a_{\xi}-\phi+\theta\right)\right. \\
\left.\left.+e^{-\beta \xi} \cos \left(p t+\alpha_{\xi}-\phi+\theta\right)\right]\right\} \\
V=\frac{E \sqrt{a^{2}+\beta^{2}}}{2 \sqrt{K_{1}^{2}+K_{2}^{2}}}\left\{\left[-e^{\beta \xi} \cos (p t-a \xi+\theta+\omega)+\right.\right. \\
\left.e^{-\beta \xi} \cos (p t+a \xi+\theta+\omega)\right]+\sqrt{H_{1}^{2}+F_{1}^{2}}[\beta \xi \cos (p t-a \xi-\phi+\theta+\omega) \\
\left.\left.+e^{-\beta \xi} \cos (p t+a \xi-\phi+\theta+\omega)\right]\right\}
\end{gathered}
$$

$$
\tan \theta=\frac{K_{1}}{K_{2}}, \tan \omega=\frac{H_{1}}{F_{1}}, \tan a=\frac{\beta}{a}
$$

$K_{1}$ and $K_{2}$ are given by equations (I 7 ) or (I8).

$H_{1}$ and $F_{1}$ are given by equations (IO).

$a$ and $\beta$ are given by equations $(7)$.

$$
H_{1}^{2}+F_{1}^{2}=\frac{C^{2} p^{2}}{a^{2}+\beta^{2}}\left\{\left(L_{1} p-\frac{I}{C_{1} p}\right)^{2}+R^{2}\right\}
$$

If we neglect the sending apparatus, then $K_{1}^{2}+K_{2}^{2}$ will reduce to the following:

$$
\begin{gathered}
K_{1}^{2}+K_{2}^{2}=\frac{e^{2 \beta l}+e^{-2 \beta l}}{4}\left\{\left(a^{2}+\beta^{2}\right)+C^{2} p^{2}\left(R_{1}^{2}+\left(L_{1} p-\frac{I}{C_{1} p}\right)^{2}\right)\right\} \\
-\frac{I}{2} \cos 2 a l\left\{\left(a^{2}+\beta^{2}\right)-C^{2} p^{2}\left(R_{1}^{2}+\left(L_{1} p-\frac{I}{C_{1} p}\right)^{2}\right)\right\}+C p \sin a l \\
\left\{a\left(L_{1} p-\frac{I}{C_{1} p}\right)-\beta R_{1}\right\}-\frac{e^{2 \beta l}-e^{-2 \beta l}}{2}\left\{\beta C p\left(L_{1} p-\frac{I}{C_{1} p}\right)+a C p R_{1}\right\}
\end{gathered}
$$


Equations (I9) and (20) are the most complete and general expressions for the current and potential at any point on the line, and take into consideration the electrical constants (resistance, inductance, capacity) of the sending and receiving apparatus. An examination of equation (I9) will show that we have four distinct waves traveling on the line. The first part represents two waves oppositely directed, sent out by transmitting apparatus, and the second part represents the reflected waves. It is quite evident that the reflected wave will not affect the receiver, and this appears from equation (I9); when $s=l, \xi=0$, and equation (I9) reduces to

$$
X_{l}=\frac{E C p \cos (p t+\theta)}{\sqrt{K_{1}^{2}+K_{2}^{2}}}
$$

A similar condition obtains for the potential, except that in this case it is the second part of equation (2O) that represents the part of the wave absorbed by the receiving instrument, for it is evident that the drop on the receiver must be proportional to the impedance of the instrument, which is the factor $\sqrt{H_{1}^{2}+F_{1}^{2}}$, and thus when $s=l$ we have

$$
V_{1}=E \frac{\sqrt{a^{2}+\beta^{2}} \sqrt{H_{1}^{2}+F_{1}^{2}}}{\sqrt{K_{1}^{2}+K_{2}^{2}}} \cos (p t-\varphi+\theta+\omega)
$$

Introducing the value of $\sqrt{H_{1}^{2}+F_{1}^{2}}$ from equation (2I) we get :

$$
\mathrm{V}_{1}=\frac{E C p \sqrt{\left(L_{1} p-\frac{\mathrm{I}}{c_{1} p}\right)^{2}+R_{1}^{2}}}{\sqrt{K_{1}^{2}+K_{2}^{2}}} \cos (p t-\phi+\theta+\omega)
$$

For the purpose of this paper it will be sufficient to consider equation (23). Suppose we calculate the maximum value of $X$, that is $\frac{C P}{\sqrt{K_{1}^{2}+K_{2}^{2}}}$ for several different frequencies, first by neglecting the terminal apparatus, and second by taking into account the terminal apparatus; then evidently the ratio of the values as calculated for the different frequencies will be a measure of the distortion. Now comparing these ratios for the different frequencies with and without the terminal apparatus will evidently give us a measure of the effect produced by the terminal apparatus. 
In my calculations I have neglected the transmitting apparatus and considered only the effect of the receiving apparatus on the distortion. This reduces the labor of computation very materially, and at the same time shows just as clearly the effect of any terminal apparatus on distortion.

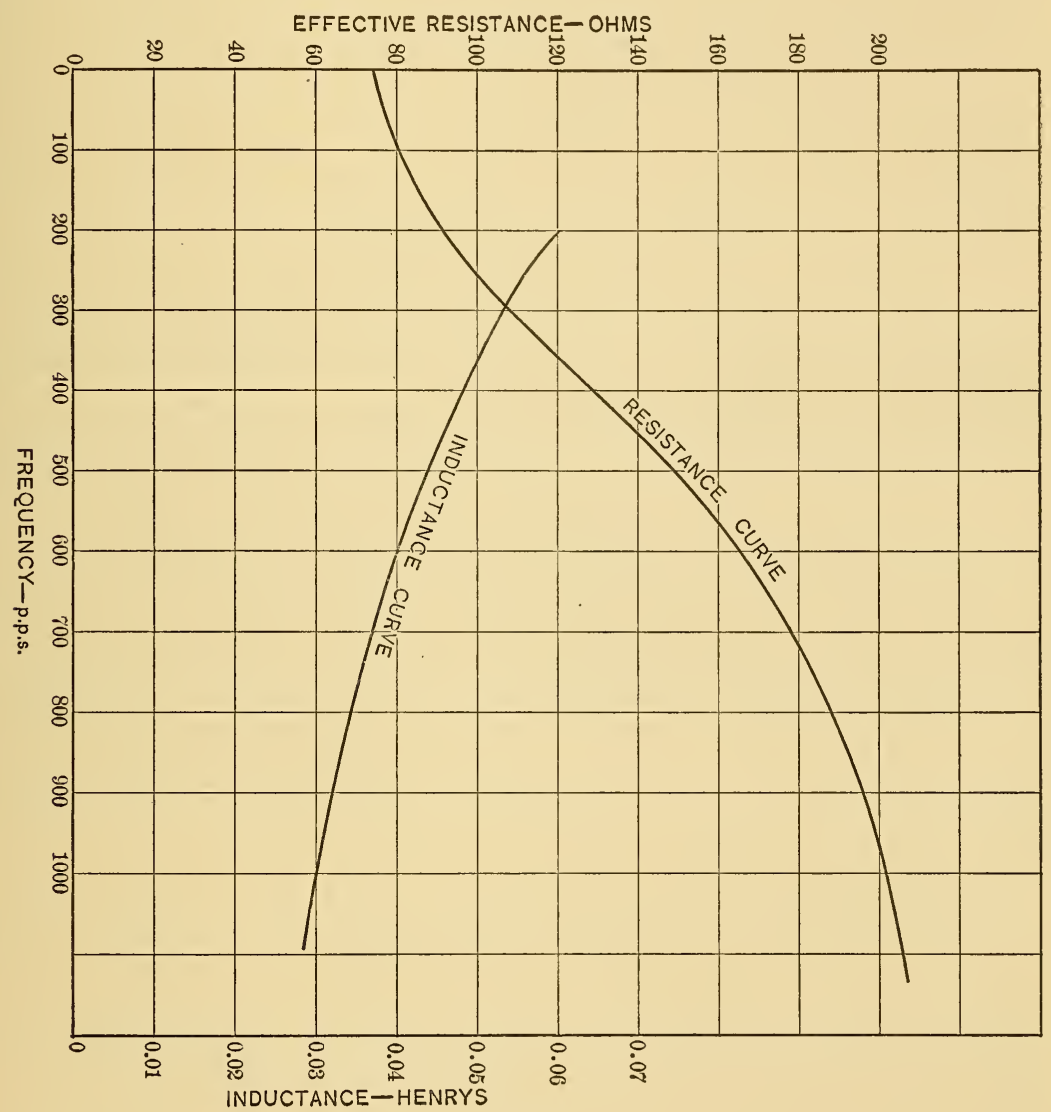

Fig. 1.

In computing the value of $X_{l}$ as given by equation (23) it must be remembered that the inductance and resistance of the receiver are functions of the frequencies, and I an indebted to Mr. C. E. Scribner, of the Western Electric Company, for furnishing me curves showing the relation of the resistance and inductance with the frequency of the receiver manufactured by the Western Electric Company. The curves are given in Fig. (I). 
In calculating the value of $X$ as given by the above equation I have assumed three distinct cases:

(I) The terminal apparatus neglected entirely;

(2) The receiver included in circuit;

(3) The receiver in series with a condenser in circuit.

I have also assumed three distinct frequencies, 500, Iooo, and I500, and two different lines, one $30 \mathrm{~km}$ long and one $300 \mathrm{~km}$ long.

The constants of $30-\mathrm{km}$ line (cable, I9 B. S.) are approximately as follows:

$L=0.0000$ I henry per $\mathrm{km}$.

$C=0.045$ microfarad per $\mathrm{km}$.

$R=27.5$ ohins per $\mathrm{km}$.

Using these constants and assuming that the impressed voltage corresponding to the frequencies 500 , I000, I500 are $E_{1}, E_{3}, E_{5}$, respectively, we shall have the following table:

TABLE I.

\begin{tabular}{l|c|c|c}
\hline & Frequency $=500$ & Frequency $=1000$ & Frequency $=1500$ \\
\cline { 1 - 2 } $\begin{array}{c}\text { Case 1- } \\
\text { No terminal apparatus .... }\end{array}$ & $X_{l}=0.00114 \mathrm{E}_{1}$ & $X_{l}=0.00099 \mathrm{E}_{3}$ & $X_{l}=0.00089 \mathrm{E}_{5}$ \\
$\begin{array}{r}\text { Case 2- } \\
\text { Receiver in circuit ........ }\end{array}$ & $X_{l}=0.00175 \mathrm{E}_{1}$ & $X_{l}=0.005 \mathrm{E}_{3}$ & $X_{l}=0.0046 \mathrm{E}_{5}$ \\
$\begin{array}{r}\text { Case 3- } \\
\text { Receiver and a condenser of } \\
1.5 \mathrm{mf} \text { in circuit ......... }\end{array}$ & $X_{l}=0.0018 \mathrm{E}_{1}$ & $X_{l}=0.0019 \mathrm{E}_{3}$ & $X_{l}=0.0045 \mathrm{E}_{5}$ \\
\hline
\end{tabular}

An examination of the above table shows that the receiver in the circuit increases the distortion, and it is further seen that the introduction of a condenser will improve the transmission.

Now while telephoning at such short distance is possible with almost any apparatus, yet it will be admitted that there is considerable room for improvement, and it would seem that the introduction of a condenser of the proper magnitude in series with the receiver ought to produce good results.

To show the effect on the distortion in long distance transmission I have made similar calculations for a cable (I 3 B. S.) $300 \mathrm{~km}$ long. 
The electrical constants of such a cable are approximately as follows:

$$
\begin{aligned}
& L=0.000 I \text { henry per } \mathrm{km} \\
& C=0.045 \text { microfarad per } \mathrm{km} \\
& R=7 \text { ohms per } \mathrm{km} .
\end{aligned}
$$

Using these values and the constants of the receiver the same as in the previous case, we get the following table:

TABLE II.

\begin{tabular}{l|c|c|c}
\hline & Frequency $=500$ & Frequency $=1000$ & Frequency $=1500$ \\
\hline $\begin{array}{c}\text { Case 1-- } \\
\text { No terminal apparatus ..... }\end{array}$ & $X_{l}=0.003 \mathrm{E}_{1}$ & $X_{l}=0.00011 \mathrm{E}_{3}$ & $X_{l}=0.000053 \mathrm{E}_{5}$ \\
$\begin{array}{c}\text { Case 2- } \\
\text { Receiver in circuit ......... }\end{array}$ & $X_{l}=0.00126 \mathrm{E}_{1}$ & $X_{l}=0.00017 \mathrm{E}_{3}$ & $X_{l}=0.00004 \mathrm{E}_{5}$ \\
$\begin{array}{c}\text { Case 3- } \\
\text { Receiver and a condenser } n f \\
1.5 \mathrm{mf} \text { in circuit ......... }\end{array}$ & $X_{l}=0.0014 \mathrm{E}_{1}$ & $X_{l}=0.0002 \mathrm{E}_{3}$ & $X_{l}=0.00005 \mathrm{E}_{5}$ \\
\hline
\end{tabular}

From the table it can be seen that the introduction of the telephone receiver does not materially influence telephonic transmission.

WashingTon, August 3, I908. 